\title{
Successful Treatment with Alectinib for Choroidal Metastasis in Anaplastic Lymphoma Kinase Rearranged Non-small Cell Lung Cancer
}

\author{
Tomoko Funazo ${ }^{1}$, Kyohei Morita ${ }^{2}$, Naoya Ikegami $^{2}$, Chisato Konishi ${ }^{2}$, Satoshi Nakao ${ }^{2}$, \\ Ryo Ariyasu ${ }^{2}$, Masato Taki ${ }^{2}$, Kazuhiko Nakagawa ${ }^{2}$, Moon Hee Hwang ${ }^{2}$, Chie Yoshimura ${ }^{2}$, \\ Toshiaki Wakayama ${ }^{2}$ and Yasuo Nishizaka ${ }^{2}$
}

\begin{abstract}
:
Choroidal metastasis is rare in cancer patients and it may cause visual disturbances that reduce their quality of life. In non-small cell lung cancer (NSCLC), targeted therapy against actionable driver mutations has gradually replaced radiotherapy as the treatment of choice for choroidal metastasis. Recently, there have been several case reports of choroidal metastasis in patients with anaplastic lymphoma kinase (ALK)-rearranged NSCLC. We herein report the case of a 40-year-old Japanese woman diagnosed with choroidal metastasis of an ALK-rearranged NSCLC who received alectinib as the first-line chemotherapy. Alectinib may be the best treatment for choroidal metastasis in patients harboring an ALK translocation because of its favorable side effect profile involving visual disturbances.
\end{abstract}

Key words: choroidal metastasis, anaplastic lymphoma kinase (ALK), alectinib, non-small cell lung cancer (NSCLC)

(Intern Med 56: 2317-2320, 2017)

(DOI: 10.2169/internalmedicine.8488-16)

\section{Introduction}

Choroidal metastasis from lung cancer is relatively rare; however, lung cancer is the second most common cancer (1).

The consensus treatment for metastasis to the orbit is radiotherapy, primarily with palliative intent to improve the visual function. However, long-term complications of radiotherapy remain a concern (2).

Systemic chemotherapy has been reported to be effective against choroidal metastasis (1), and we herein report a case involving a patient with choroidal metastasis of anaplastic lymphoma kinase (ALK)-rearranged adenocarcinoma treated with alectinib as the first-line chemotherapy.

\section{Case Report}

A 40-year-old Japanese woman with a smoking history of one pack year presented with a 1-month history of photopsia in her right eye. Her best corrected visual acuity (BCVA) was $1.5(\log M A R-0.2)$ at the first visit and it decreased to 0.3 (logMAR 0.5) after 1 month. A fundus examination of her right eye (Fig. 1) revealed an elevated lesion with exudative retinal detachment that involved the optic disc. Fluorescein angiography showed multiple hyperfluorescent spots from areas of choroid elevation with late-phase leakage. An ocular evaluation by optical coherence tomography (OCT, Fig. 2) revealed a solitary metastatic choroid tumor. Thorax computed tomography (CT, Fig. 3) revealed a nodule in the upper lobe of the left lung and no mammary abnormalities. She had hepatic and bone metastases. Poorly differentiated adenocarcinoma of the lung was diagnosed by a transbron-

${ }^{1}$ Department of Respiratory Medicine, Kyoto University, Japan and ${ }^{2}$ Department of Respiratory Medicine, Osaka Red Cross Hospital, Japan Received: November 1, 2016; Accepted: December 27, 2016; Advance Publication by J-STAGE: August 10, 2017 Correspondence to Dr. Tomoko Funazo, funazo@kuhp.kyoto-u.ac.jp 


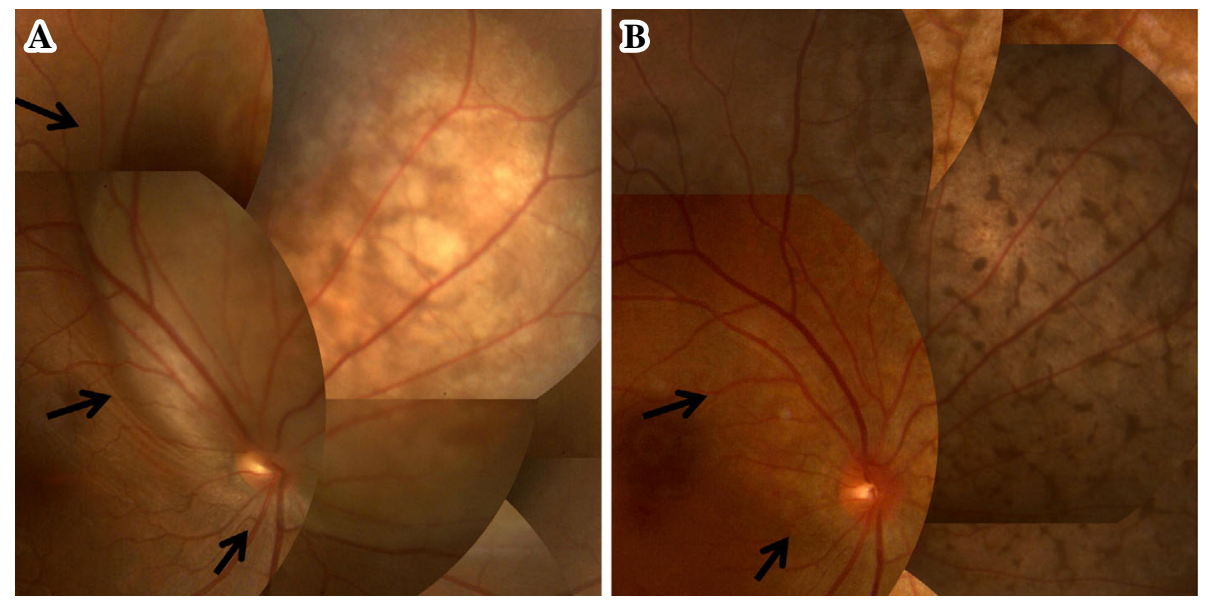

Figure 1. (A) Pretreatment fundus photograph of the right eye showing a severe elevated subretinal lesion. (B) Fundus photograph of right eye 2 months after starting alectinib treatment showing the subretinal lesion to have significantly decreased in size (arrow).
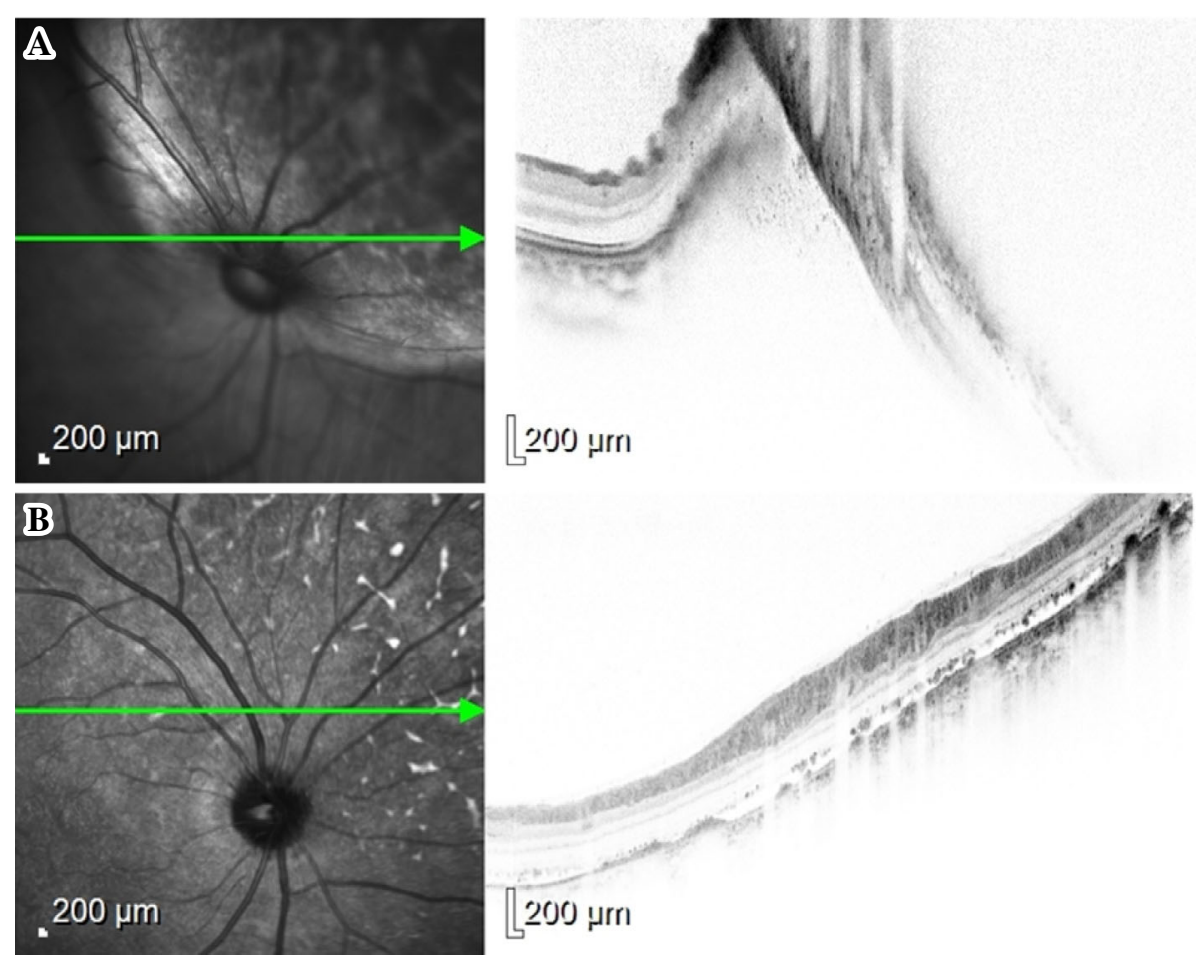

Figure 2. (A) Pretreatment optical coherence tomography (OCT) of the right eye during the prealectinib screening ophthalmologic examination showing serous retinal detachment and an elevation of the retinal epithelial layer because of the presence of tumor. (B) OCT of the right eye after 2 months of alectinib treatment showing a resolution of the subretinal fluid.

chial biopsy. Genetic testing found her negative for epidermal growth factor receptor (EGFR) mutation. However, immunohistochemical staining for ALK gene rearrangement showed extensive positivity at the primary pulmonary site (Fig. 4) and florescent in situ hybridization demonstrated $54 \%$ positivity for echinoderm microtubule-associated protein-like 4 and ALK gene translocation.

She started treatment with alectinib which was a second generation ALK tyrosine kinase inhibitor. The pulmonary nodule and liver metastasis decreased in size after 2 months and were assessed as partial response according to the re- sponse evaluation criteria of solid tumors. Posttreatment BCVA improved from 0.3 to 0.8 (logMAR 0.5 to 0.1 ) after 5 months. She experienced some side effects, such as slight liver dysfunction and a rash, but both resolved within a few days. Despite the response of choroidal lesion, alectinib was discontinued after 9 months because of disease progression with new multiple bone metastases. The cytotoxic chemotherapy, comprising cisplatin, pemetrexed, and bevasizumab, was effective for back pain from bone metastasis; her symptomatic improvement has continued at the time of writing this report (15 months). 

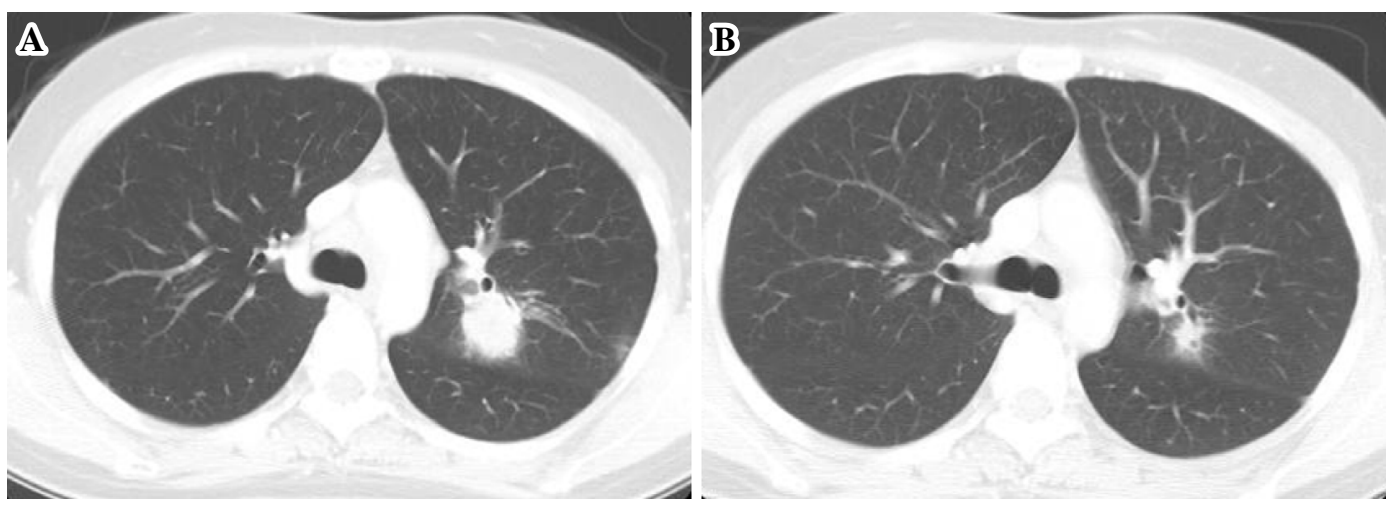

Figure 3. (A) A pretreatment CT scan before the start of alectinib treatment, (B) A CT scan 2 months after starting alectinib treatment demonstrating a partial response of the tumor.

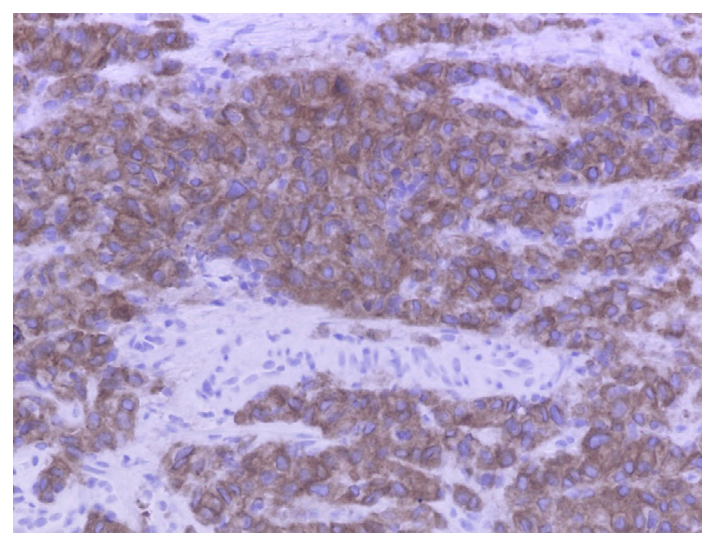

Figure 4. Immunohistochemical staining for ALK gene rearrangement using anti-ALK antibody showing extensive positivity in a lung biopsy specimen. ALK: anaplastic lymphoma kinase

\section{Discussion}

Ocular metastasis occurs in $2-9 \%$ of all malignancies; $47-$ $81 \%$ are from breast cancer and $9-23 \%$ from lung cancer (3). Most intraocular metastases localize to the choroid, which has the most developed vasculature and highest blood flow of the ocular tissues (4). Metastatic ocular lesions may be clinically silent; nevertheless, the majority of choroidal metastases are diagnosed before the diagnosis of primary lung cancer (5). A recent meta-analysis of 55 cases of choroidal metastasis of lung cancer reported the mean age of the patients to be 55 years; $42 \%$ of the primary tumors were adenocarcinomas; and $22 \%$ of the patients were neversmokers (1).

The standard treatment for choroidal metastases is ocular radiotherapy or photocoagulation intended to preserve vision $(2,3,6,7)$; however, it is associated with various complications, such as cataracts $(0.04 \%)$, retinopathy, and glaucoma $(0.01 \%)$. As the duration before the complications of radiation start to occur (6 months) is greater than the median survival period of choroidal metastasis from non-small cell lung cancer (NSCLC) (5.2 months) (8), the complica- tions of the former have not yet been considered to be significant. As molecular targeted therapy has resulted in an improved survival, targeted therapy for choroidal metastasis is thus considered to be superior to radiotherapy. It is a key step to confirm the presence of an oncogene mutation as soon as possible to avoid any unnecessary radiotherapy.

ALK-rearranged NSCLC is a rare subtype of oncogenic driver, accounting for approximately $5 \%$ of all cases, and it is mostly mutually exclusive to EGFR mutations (9). An ALK gene rearrangement is more frequent in young patients who were never-smokers, and in adenocarcinomas $(10,11)$. These two characteristics - young and adenocarcinoma - are closely related to choroidal metastasis of NSCLC. The relationship between the mechanisms of choroidal metastasis and ALK-rearranged NSCLC is unknown. However, this hypothesis warrants future studies for confirmation.

The available targeted therapies for advanced ALKrearranged NSCLC have higher response rates and longer progression-free survival (PFS) than cytotoxic chemotherapy. They are crizotinib, a first generation ALK inhibitor (10), and alectinib (12) and ceritinib (13), which are second generation inhibitors. There have been five case reports of patients with choroidal metastasis of ALK-rearranged NSCLC (14-18). These four case reports were based on crizotinib therapy, and one case report was based on alectinib therapy for a crizotinib-resistant patient.

We believe that this is first reported patient given alectinib as first-line chemotherapy. The National Comprehensive Cancer Network guidelines recommend crizotinib as a firstline chemotherapy for ALK-rearranged NSCLCs at the time of diagnosis. However, crizotinib is associated with some side effects. One of them is vision disorder. In a randomized phase III in ALK rearranged NSCLC patients, visual disturbance occurred in $55 \%$ patients received crizotinib treatment, but only occurred in $1 \%$ of patients received alectinib treatment (19). Our patient presented with poor vision in the right eye, and to avoid the possible risk of any additional vision disturbance, we selected alectinib.

In summary, detecting an oncogenic driver is an important step in delivering the best available patient care. In our patient with choroidal metastasis of ALK-rearranged adenocar- 
cinoma, alectinib was chosen over crizotinib because of the side effects profile of the latter.

The authors state that they have no Conflict of Interest (COI).

\section{Acknowledgement}

The authors would like to thank Enago (www.enago.jp) for the English language review.

\section{References}

1. Singh N, Kulkarni P, Aggarwal AN, et al. Choroidal metastasis as a presenting manifestation of lung cancer: a report of 3 cases and systematic review of the literature. Med (United States) 91: 179194, 2012.

2. Jardel P, Sauerwein W, Olivier T, et al. Management of choroidal metastases. Cancer Treat Rev 40: 1119-1128, 2014.

3. Kanthan GL, Jayamohan J, Yip D, Conway RM. Management of metastatic carcinoma of the uveal tract: an evidence-based analysis. Clin Exp Ophthalmol 35: 553-565, 2007.

4. Cunha-Vaz JG, Shakib M, Ashton N. Studies on the permeability of the blood-retinal barrier. I. On the existence, development, and site of a blood-retinal barrier. Br J Ophthalmol 50: 441-453, 1966.

5. Shields CL, Shields J a, Gross NE, Schwartz GP, Lally SE. Survey of 520 eyes with uveal metastases. Ophthalmology 104: 12651276, 1997.

6. Mauget-Faÿsse M, Gambrella J, Quaranta-El Maftouchi M, Moullet I. Photodynamic therapy for choroidal metastasis from lung adenocarcinoma. Acta Ophthalmol Scand 84: 552-554, 2006.

7. Wiegel T, Bottke D, Kreusel KM, et al. External beam radiotherapy of choroidal metastases - final results of a prospective study of the German Cancer Society (ARO 95-08). Radiother Oncol 64: 13-18, 2002.

8. Stephens RF, Shields J a. Diagnosis and management of cancer metastatic to the uvea: a study of 70 cases. Ophthalmology 86: 1336-1349, 1979.

9. Shaw AT, Engelman J a. ALK in lung cancer: past, present, and future. J Clin Oncol 31: 1105-1111, 2013.

10. Shaw AT, Kim DW, Nakagawa K, et al. Crizotinib versus chemo- therapy in advanced ALK-positive lung cancer. N Engl J Med 368: 2385-2394, 2013.

11. Popat S, Gonzalez D, Min T, et al. ALK translocation is associated with ALK immunoreactivity and extensive signet-ring morphology in primary lung adenocarcinoma. Lung Cancer 75: 300305, 2012.

12. Seto T, Kiura K, Nishio M, et al. CH5424802 (RO5424802) for patients with ALK-rearranged advanced non-small-cell lung cancer (AF-001JP study): a single-arm, open-label, phase 1-2 study. Lancet Oncol 14: 590-598, 2013.

13. Shaw AT, Kim D-W, Mehra R, et al. Ceritinib in ALK-rearranged non-small-cell lung cancer. N Engl J Med 370: 1189-1197, 2014.

14. Jiang K, Brownstein S, Sekhon HS, et al. Ocular metastasis of lung adenocarcinoma with ELM4-ALK translocation: a case report with a review of the literature. Saudi J Ophthalmol 27: 187-192, 2013.

15. Feng Y, Singh AD, Lanigan C. Choroidal metastases responsive to crizotinib therapy in a lung adenocarcinoma patient with ALK 2p23 fusion identified by ALK immunohistochemistry. J Thorac Oncol 8: e109-e111, 2013.

16. Rao RC, Gragoudas ES. Choroidal metastases from EML4-ALKpositive non-small-cell lung adenocarcinoma. J Clin Oncol 33: e112-e114, 2015

17. Bearz A, Santarossa S, Manfrè A, et al. Activity of crizotinib over cerebral and choroidal metastases in Non-Small-Cell Lung Cancer (NSCLC)-ALK rearranged: a case report. BMC Res Notes 7: 589, 2014.

18. Okuma Y, Tanaka Y, Kamei T, Hosomi Y, Okamura T. Alectinib for choroidal metastasis in a patient with crizotinib-resistant ALK rearranged positive non-small cell lung cancer. Onco Targets Ther 8: 1321-1325, 2015.

19. Hida $\mathrm{T}$, Nokihara $\mathrm{H}$, Kondo $\mathrm{M}$, et al. Alectinib versus crizotinib in patients with ALK-positive non-small-cell lung cancer (J-ALEX): an open-label, randomised phase 3 trial. Lancet 6736: 1-11, 2017 [Epub ahead of print].

The Internal Medicine is an Open Access article distributed under the Creative Commons Attribution-NonCommercial-NoDerivatives 4.0 International License. To view the details of this license, please visit (https://creativecommons.org/licenses/ by-nc-nd/4.0/).

(C) 2017 The Japanese Society of Internal Medicine Intern Med 56: 2317-2320, 2017 